\title{
Endoscopic retrograde cholangiopancreatography through a gastric stoma using an ultrathin endoscope: a novel approach
}

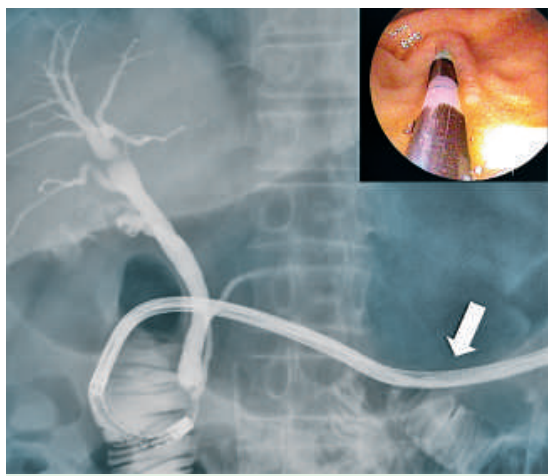

Fig. 1 Cholangiopancreatography was performed using an ultrathin transnasal endoscope in patient 1 . Note that the scope was inserted through the gastric stoma (arrow). Inset: Endoscopic view of the papilla of Vater.

Because a transoral or transnasal approach is essential to the performance of endoscopic retrograde cholangiopancreatography (ERCP) [1], difficulties are often encountered in complicated cases when either approach is problematic, including in patients who have suffered a cerebrovascular accident. In such patients percutaneous endoscopic gastrostomy (PEG) has often previously been carried out for long-term nutritional management. Here we describe the successful performance of ERCP through the gastric stoma using an ultrathin forward-viewing endoscope (EG530N5; Fujinon-Toshiba, Tokyo, Japan).
We have carried out trans-stomal ERCP in two patients with a PEG, who had dementia due to cerebral infarction. Patient 1 was an 81-year-old man referred to our hospital for suspected cholecystitis, with a swollen gallbladder seen on computed tomography (CT). We performed ERCP via the PEG stoma to avoid the risk of aspiration pneumonia, and confirmed that there was no obstruction or lithiasis in the biliary tract ( $\bullet$ Fig. 1 ).

Patient 2 was an 89-year-old woman referred to us for jaundice, with elevated serum levels of bilirubin $(5.0 \mathrm{mg} / \mathrm{dl})$, alkaline phosphatase (968 IU/1) and C-reactive protein $(4.1 \mathrm{mg} / \mathrm{dl})$. Abdominal CT showed dilatation of both gallbladder and common bile duct (CBD). We carried out trans-stomal ERCP in this patient also, and diagnosed a stricture in the lower CBD without malignant findings or lithiasis ( $\bullet$ Fig. 2a). Her laboratory results were rapidly restored to normal levels after placement of a drainage tube in the CBD ( $\bullet$ Fig. $2 \mathbf{b})$.

In both cases there was no difficulty in identifying the papilla of Vater ( $\bullet$ Fig. 1 and $\odot 2$, insets) or in cannulation for cholangiopancreatography using the conventional ERCP tube through the biopsy channel. No significant complications were encountered, including cardiopulmonary damage or post-procedural hyperamylasemia.
To our knowledge, this is the first report describing performance of ERCP through a gastric stomal, using an ultrathin endoscope, and which demonstrates its benefit and tolerability. Because the procedure does not require a special technique for controlling the endoscope, this new method can be widely applied in patients with a gastric stoma.

Endoscopy_UCTN_Code_TTT_1AR_2AJ Endoscopy_UCTN_Code_TTT_1AO_2AK

\section{A. Mori, N. Ohashi, T. Maruyama, \\ H. Tatebe, K. Sakai, H. Inoue, \\ S. Takegoshi, M. Okuno}

Department of Gastroenterology, Inuyama Chuo Hospital, Aichi, Japan

\section{References}

1 Mori A, Asano T, Maruyama T et al. Transnasal ERCP/ENBD using an ultrathin esophagogastroduodenoscope. J Gastroenterol 2006; 41: $1237-1238$

\section{Bibliography}

DOI 10.1055/s-2007-966804

Endoscopy 2007; 39: E323

(c) Georg Thieme Verlag KG Stuttgart · New York . ISSN 0013-726X

\section{Corresponding author}

\section{A. Mori, MD}

Department of Gastroenterology

Inuyama Chuo Hospital

Aichi 484-8511

Japan

Fax: + 81-568-628761

a-mori@inuyamachuohospital.or.jp
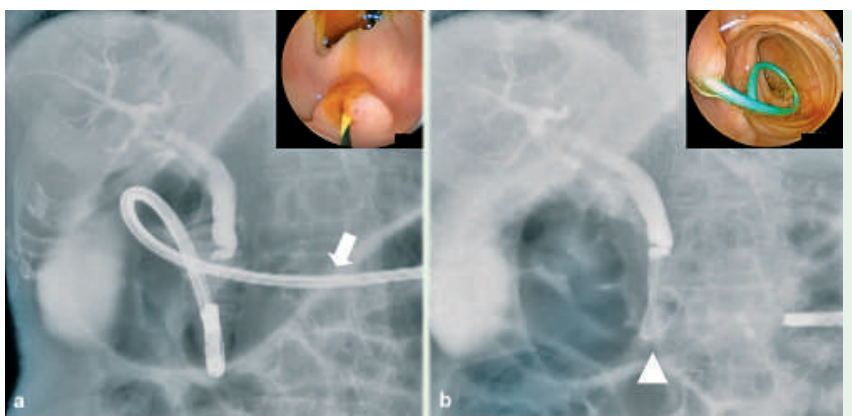

Fig. 2 a Cholangiopancreatography performed through the gastric stoma (arrow) in patient 2. Inset: Endoscopic view of papilla of Vater. b A drainage tube was inserted into the common bile duct (arrowhead). 